\title{
Empleo de benzodiacepinas y riesgo de demencia o deterioro cognitivo
}

Benzodiazepine use and risk of incident dementia or cognitive decline

Gray SL. y col. BMJ 2016;352:1-9.

\section{Objetivos}

Determinar si el uso acumulado de benzodiacepinas está asociado con un mayor riesgo de demencia o una más rápida declinación cognitiva.

\section{Diseño, lugar y pacientes}

Estudio de cohorte prospectivo realizado en Seattle, Estados Unidos, en un sistema de salud integrado sobre pacientes $\geq$ de 65 años, sin demencia. Los participantes se reclutaron desde 1994 hasta 2004; fueron evaluados al inicio del estudio y posteriormente cada dos años, por un total de siete años, para determinar la función cognitiva y recoger las características demográficas, la historia médica y el estado de salud. Se analizaron los participantes que tenían al menos diez años de afiliación al sistema de salud para asegurar datos suficientes sobre la exposición a las BDZ a largo plazo.

Para la detección de demencia y enfermedad de Alzheimer, se utilizó un instrumento de cribado de capacidades cognitivas (Cognitive Abilities Screening Instrument [CASI])1. Lo mismo se utilizó para evaluar la trayectoria cognitiva. El diagnóstico de demencia y de enfermedad de Alzheimer se hicieron con los criterios diagnósticos estándar.

\section{Evaluación de la exposición a las benzodiacepinas}

La exposición a las benzodiacepinas se definió a partir de datos computarizados de farmacia. Para crear las medidas de exposición, se calculó una dosis diaria estandarizada (DDE) dividiendo el producto total consumido por la dosis mínima efectiva por día que se recomienda en adultos mayores. Para cada participante se calcularon las dosis totales estandarizadas (DTE) que consistieron en las DDE suministradas durante un período de diez años. El año previo al inicio del estudio fue excluido debido a su posible uso para los síntomas prodrómicos de demencia. El uso acumulativo de benzodiacepinas se clasificó en 1 a 30, 31 a 120, o $\geq 121$ DTE basados en la distribución de la exposición.

\section{Resultados}

Se incluyeron 3.434 participantes en el análisis, que correspondieron a 25.019 personas-año de seguimiento, con una media de 7,3 (DE 4,8) años. La mediana de edad fue 74,4 (rango intercuartilo 70 a 80$)$.

Las benzodiacepinas utilizadas con mayor frecuencia fueron temazepam, diazepam, clonazepam, triazolam, y lorazepam, que en conjunto representaron el $83 \%$ de la exposición a este grupo de fármacos.

Dentro de la categoría más alta de DTE de benzodiacepinas ( $\geq 121$ DTE), el nivel medio de utilización fue de 375 DTE (equivalente a un poco más de un año de uso diario).

Un total de 797 pacientes $(23,2 \%)$ desarrollaron demencia durante el seguimiento, de los cuales $637(79,9 \%)$ presentaron enfermedad de Alzheimer. Ver tabla 1.

Tabla 1. Riesgo de desarrollar demencia y enfermedad de Alzheimer para cada nivel de exposición acumulada de benzodiacepinas comparado con el no uso.

\begin{tabular}{|c|c|c|c|}
\hline & $\begin{array}{l}\text { DTE } \pm 1 \text { a } 30 \\
\text { HR* (IC 95\%) }^{*}\end{array}$ & $\begin{array}{l}\text { DTE } \pm 31 \text { a } 120 \\
\text { HR* }^{\star} \text { (IC 95\%) }\end{array}$ & $\begin{array}{c}\text { DTE } \geq 121 \\
\mathrm{HR}^{*} \text { (IC 95\%) }\end{array}$ \\
\hline Demencia & $1,25(1,03$ a 1,51$)$ & 1,31 (1 a 1,71) & $1,07(0,82$ a 1,39$)$ \\
\hline Enfermedad de Alzheimer & $1,27(1,03$ a 1,57$)$ & $1,16(0,84$ a 1,60$)$ & $0,95(0,71$ a 1,27$)$ \\
\hline
\end{tabular}

„DTE: Dosis total estandarizada *Hazard ratio ajustado por edad, sexo, nivel educativo, hipertensión, diabetes mellitus, tabaquismo, accidentes cerebrovasculares, cardiopatía coronaria, índice de masa corporal, ejercicio regular, autopercepción de la salud, y síntomas de depresión.

\section{Conclusión}

El riesgo de demencia es ligeramente mayor en personas con mínima exposición a benzodiacepinas pero no con los mayores niveles de exposición. Estos resultados no avalan una asociación causal entre la utilización de benzodiacepinas y el desarrollo de demencia.

\section{Comentario}

Las benzodiacepinas se asocian con muchos efectos nocivos: caídas, fracturas, accidentes de tránsito, y delirium. Debido a estos riesgos, las benzodiacepinas no están recomendadas para el tratamiento del insomnio, agitación o delirio en los adultos mayores, y se recomienda que en caso de utilizarlas, sea por corto tiempo ${ }^{2}$. No obstante, el uso de benzodiacepinas aumenta con la edad y los adultos mayores las utilizan por largo tiempo'. Se ha tratado de encontrar una relación causal entre el uso de benzodiacepinas y el aumento de demencia. Es todo un desafío discernir si el uso de BDZ constituye un factor de riesgo o simplemente es un confundidor dada su frecuente indicación para los síntomas neuropsiquiátricos como insomnio, ansiedad y depresión, que preceden la instalación del deterioro cognitivo.

\section{Conclusiones del comentador}

A pesar de que este estudio sugiere que el uso de benzodiacepinas no aumenta el riesgo de demencia o deterioro cognitivo en adultos mayores, se recomienda evitar igualmente su uso en esta población a fin de prevenir la aparición de efectos adversos bien documentados.

María Elena Guajardo [ Servicio de Clínica Médica del Hospital Italiano de Buenos Aires maria.guajardo@hospitalitaliano.org.ar ]

Guajardo ME. Empleo de benzodiacepinas y riesgo de demencia o deterioro cognitivo. Evid Act Pract Ambul. 2017;20(2):55. Comentado de: Gray SL, y col. Benzodiazepine use and risk of incident dementia or cognitive decline: prospective population based study. BMJ. 2016;352:i90. PMID: 26837813

Referencias bibliográficas

1. Teng EL, y col. The Cognitive Abilities Screening Instrument (CASI): a practical test for cross-cultural epidemiological studies of dementia. Int Psychogeriatr $1994 ; 6: 45-58$. 2. Gallacher J, y col. Benzodiazepine use and risk of dementia: evidence from the Caerphilly Prospective Study (CaPS). J Epidemiol Community Health 2012;66:869-73. doi:10.1136/jech-2011-200314 